\title{
REMARQUES COMPLÉMENTAIRES ET ÉTUDE COMPARATIVE \\ DES TRAVAUX RELATIFS AUX PERTES DANS LES LABYRINTHES \\ CYLINDRIQUES LISSES
}

PARTIE

\section{PAR R. HUGUENIN *
P. BOUILLAUD ** ET F. BREUIL * *}

Sont présentés successivement :

- les notations;

- les caractéristiques des joints utilisés par les différents expérimentateurs (MM. Viano, Petermann et Société Franco-Tosi);

- les facteurs influençant le débit de fuite (une analyse particulière est effectuée sur un facteur négligé par les expérimentateurs et très difficile à contrôler : savoir l'excentricité du joint);

- les schémas de l'écoulement dans le joint pris en considération;

- une synthèse et une discussion des travaux de M. Viano;

- une synthèse et une discussion des travaux de M. Petermann;

- une superposition des résultats obtenus par MM. Viano et Petermann;

- une superposition des résultats obtenus par $M$. Viano et la Société Franco-Tosi;

— des conclusions.

\section{Notations}

Afin de faciliter la compréhension du présent document, nous utiliserons les mêmes notations que celles utilisées dans le rapport du groupe de travail de M. Viano.

Ainsi, nous transposerons toutes les notations de la littérature incluses dans notre étude.

\section{Caractéristiques géométriques:}

Diamètre du tambour ou de la roue....... D $(\mathrm{mm})$

Longueur du labyrinthe............ L $(\mathrm{mm})$

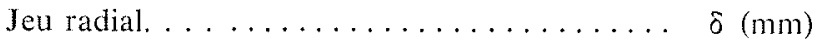

Excentricité. . . . . . . . . . . . . a $(\mathrm{mm})$

Excentricité relative. . . .......... e $=\alpha / \delta$

Caractéristiques dynamiques:

Vitesse de rotation du tambour ou de la roue. $N(t r / m n)$

Vitesse périphérique (ou tangentielle). . ... $u(\mathrm{~m} / \mathrm{s})$

Vitesse axiale moyenne de l'eau......... V $(\mathrm{m} / \mathrm{s})$

Autres caractéristiques:

Chute de pression. ............. $\Delta \mathrm{H}$

(n d'eau)

Coefficient de frottement. . . .......... $\lambda$

Viscosité cinématique. . . . . . . . . $v\left(\mathrm{~m}^{2} / \mathrm{s}\right)$

Nombre de Reynolds:

- Ecoulement axial. . . .......... $\operatorname{Re}_{v}$

- Ecoulement périphérique. . . ..... $\mathrm{Re}_{u}$

\footnotetext{
* Professeur à l'Ecole Nationale Supérjeure d’Arts et Métiers de Paris.

*:: Elèves-ingénieurs à l'Ecole Nationale Supérieure d'Arts et Métiers de Paris.
}

\section{Inventaire des facteurs influençant le débit de fuite dans les machines hydrauliques}

Nous ne citerons qu'à titre de rappel les paramètres dont l'influence sur le débit de fuite a été précédemment mise en évidence.

Ce sont:

- le jeu radial qui est le facteur déterminant du débit de fuite;

- la longueur du labyrinthe;

- le diamètre de la roue (ou du tambour);

- la vitesse axiale du fluide définissant la forme de l'écoulement;

- l'état de surface du joint, dont nous ne tiendrons pas compte, car il est difficile d'en déterminer la rugosité;

- la chute de pression à travers le joint;

- les caractéristiques physiques du fluide:

- viscosité cinématique: $1,1 \cdot 10^{-6} \mathrm{~m}^{2} / \mathrm{s}$ pour l'eau à $16^{\circ} \mathrm{C}$ (M. Viano). $0,9 \cdot 10^{-6} \mathrm{~m}^{2} / \mathrm{s}$ (M. Petermann),

- température: notons à cet effet que les essais de M. Viano ont été effectués avec de l'eau à la température de $16^{\circ} \mathrm{C}$ environ (température ambiante).

Parmi les facteurs qui infuencent le débit de fuite, il faut prendre en considération la vitesse de rotation et l'excentricité.

Comparaison des caractéristiques géométriques des joints lisses industriels à celles des joints expérimentaux

Les essais réalisés pour l'évaluation du débit de fuite dans les joints lisses ont nécessité l'emploi d'un matériel expérimental possédant les caractéristiques suivantes:

\begin{tabular}{|c|c|c|c|}
\hline & $\begin{array}{c}\text { Diamètre } \\
\text { DU TAMBOUR } \\
(\mathrm{mm})\end{array}$ & $\begin{array}{c}\text { JEU RADIAL } \\
\text { (mm) }\end{array}$ & $\begin{array}{l}\text { LONGUEUR DU } \\
\text { IABYRINTHE } \\
\text { (mm) }\end{array}$ \\
\hline Riva. . . . . & 295 & $0,1 \quad$ à 0,4 & 37,5 à 150 \\
\hline Sulzer. . . . . & 150 & 0,125 à 0,5 & 65 à 195 \\
\hline M. Petermann. & $\begin{array}{r}65 \\
85 \\
110\end{array}$ & $\begin{array}{l}0,10 \\
0,15 \\
0,20\end{array}$ & 110 \\
\hline Franco Tosi. . & 245 & 0,1 à 0,31 & 80 \\
\hline
\end{tabular}

Pour donner un ordre de grandeur des caractéristiques industrielles des joints, nous avons retenu celles des pompes centrifuges et des turbines Francis. Nous les avons rassemblées dans le tableau ci-après. 
Jeux et autres paramètres géométriques des joints industriels

\begin{tabular}{|c|c|c|c|c|c|c|}
\hline $\begin{array}{c}\text { MACHINES HYDRAULIQUES } \\
\text { INDUSTRIELLES }\end{array}$ & $\begin{array}{l}\text { DIAMÈTRE } \\
\quad \text { ROUE } \\
\text { D (en } \mathrm{mm})\end{array}$ & $\begin{array}{l}\text { JEU RADIAL } \\
\delta(\mathrm{en} \mathrm{mm})\end{array}$ & $\begin{array}{l}\text { LONGUEUR } \\
\text { JOINT } \\
\mathrm{L}(\mathrm{en} \mathrm{mm})\end{array}$ & $L / 2 \delta$ & $\begin{array}{l}\text { EXCENTRICITÉ } \\
\text { izELATIVE } \\
e\end{array}$ & $\begin{array}{r}2 \delta / D \\
10^{-3}\end{array}$ \\
\hline $\begin{array}{l}\text { Pompes centrifuges (jeu minimal pour ba- } \\
\text { gues en bronze). }\end{array}$ & 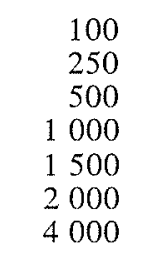 & $\begin{array}{l}0,15 \\
0,25 \\
0,285 \\
0,43 \\
0,57 \\
0,72 \\
1,71\end{array}$ & $\begin{array}{c}8 \\
15 \\
26 \\
50 \\
71,5 \\
90 \\
150\end{array}$ & $\begin{array}{l}26,7 \\
37,5 \\
45,6 \\
58,2 \\
63,0 \\
62,5 \\
43,8\end{array}$ & & $\begin{array}{l}3,0 \\
2,0 \\
1,1 \\
0,86 \\
0,76 \\
0,72 \\
0,85\end{array}$ \\
\hline Turbines Francis & $\begin{array}{r}390 \\
650 \\
1150 \\
15550 \\
2200 \\
2700 \\
35500\end{array}$ & $\begin{array}{l}0,34 \\
0,49 \\
0,70 \\
0,87 \\
1,10 \\
1,30 \\
1,50\end{array}$ & $\begin{array}{r}50 \\
70 \\
100 \\
120 \\
150 \\
170 \\
200\end{array}$ & $\begin{array}{l}73,5 \\
71,5 \\
71,5 \\
69,0 \\
68,2 \\
65,4 \\
66,7\end{array}$ & $\begin{array}{l}0,2 \\
\grave{a} \\
0,3\end{array}$ & $\begin{array}{l}1,7 \\
1,5 \\
1,2 \\
1,1 \\
1,0 \\
0,96 \\
0,86\end{array}$ \\
\hline
\end{tabular}

Pour les essais Riva et Franco Tosi, le jeu relatif $2 \delta / \mathrm{D}$ varie de 0,7 ou $0,8 \cdot 10^{-3}$ à 2,6 ou $2,7 .^{-3}$ : la majorité de la plage industrielle précédente est donc explorée. Les essais de Sulzer $\left(1,7\right.$ à $\left.6,7 \cdot 10^{-3}\right)$ et de $M$. Petermann $\left(3,1\right.$ à $\left.3,6.10^{-3}\right)$ correspondent également à un domaine industriel.

Quant au rapport $\mathrm{L} / 2 \delta$ il varie de 50 à 750 (Riva), 60 à 780 (Sulzer), 225 à 550 (M. Petermann), 130 à 400 (Franco Tosi), alors qu'il est de l'ordre de 70 pour les turbines Francis et de 30 à 60 environ pour les pompes centrifuges.

\section{Influence sur le débit de fuite de l'excentricité relative du rotor d'une machine industrielle}

Dans les pompes et turbines industrielles à axe horizontal, il est certain que le fléchissement de l'arbre et l'usure des paliers entraînent une excentration de la roue par rapport au stator.

Rapporté à de faibles jeux radiaux, ce défaut peut prendre des valeurs relatives importantes. Ainsi, l'excentricité relative $e=\alpha / \delta$ peut atteindre 0,3 .

Dans son ouvrage "Impeller Pumps», M. Adam Troskolanski trace des courbes mettant en valeur l'influence de l'excentricité.

Pour établir ces courbes (fig. II.1), il considère un joint cylindrique lisse et reporte $\lambda h=\lambda / 4(\lambda=$ coefficient de frottement) en fonction du jeu radial, pour des excentricités variables $(\alpha=0$ et $\alpha=\delta$ ).

D'après ce graphique, il apparaît que l'excentricité relative entraîne une diminution du coefficient de frottement, donc augmente le débit de fuite.

Calcul théorique du débit de fuite à travers un joint excentré :

Dans le cas d'un écoulement laminaire, en supposant que la pression est uniforme dans une section droite du joint, on peut établir que:

$$
\frac{\mathrm{Q}_{\text {excentre }}}{\mathrm{Q}_{\text {centre }}}=1+1,5 \mathrm{e}^{2} \quad(\text { avec } u=0)
$$

En régime turbulent, il est possible, par une série d'approximations successives, de déterminer la loi de variation de ce rapport qui est alors sensiblement représentée par la relation:

$$
\frac{\mathrm{Q}_{\text {excentre }}}{\mathrm{Q}_{\text {centre }}}=1+0,5 e^{2} \quad \text { (avec } u=0 \text { ). }
$$

Cette loi très approchée montre tout de même que l'excentricité influence moins le débit de fuite en régime turbulent qu'en régime laminaire. Les joints lisses des machines industrielles engendrent des écoulements de fluide en régime turbulent.

Certains essais auraient donné la relation expérimentale :

$$
\frac{Q_{\text {excentré }}}{Q_{\text {centré }}}=1+0,4 e
$$

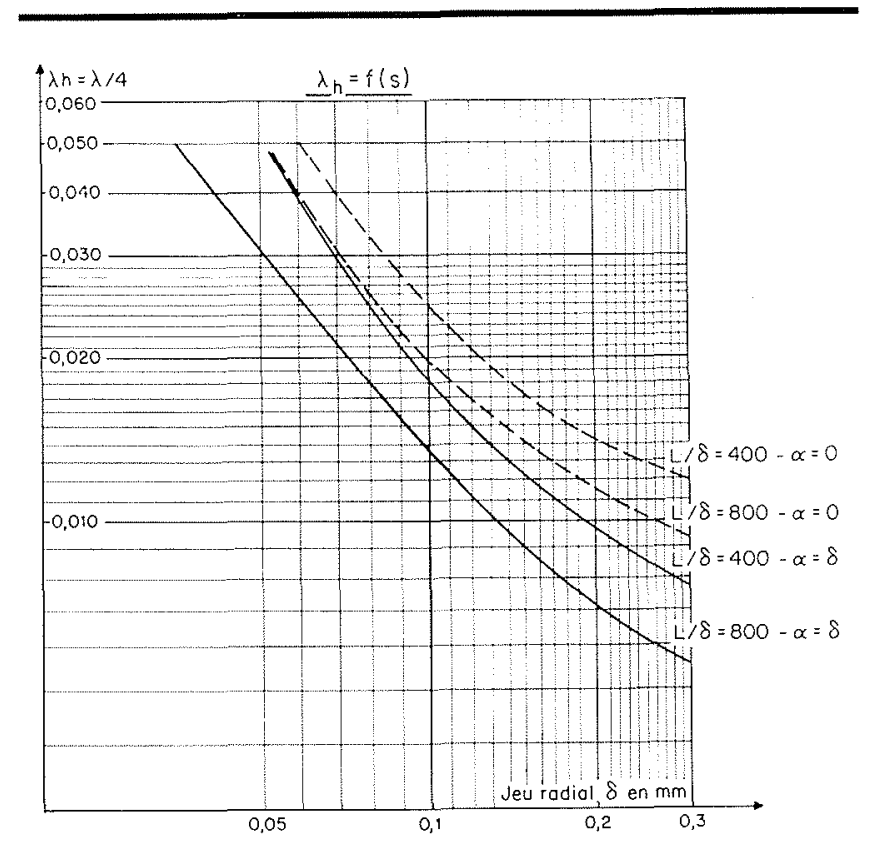

II.1/ Influence de l'excentricité relative $\alpha$ sur le coefficient de frottement $\lambda$.

Variation of friction coefficient $\lambda$ with relative eccentricity $\alpha$. 


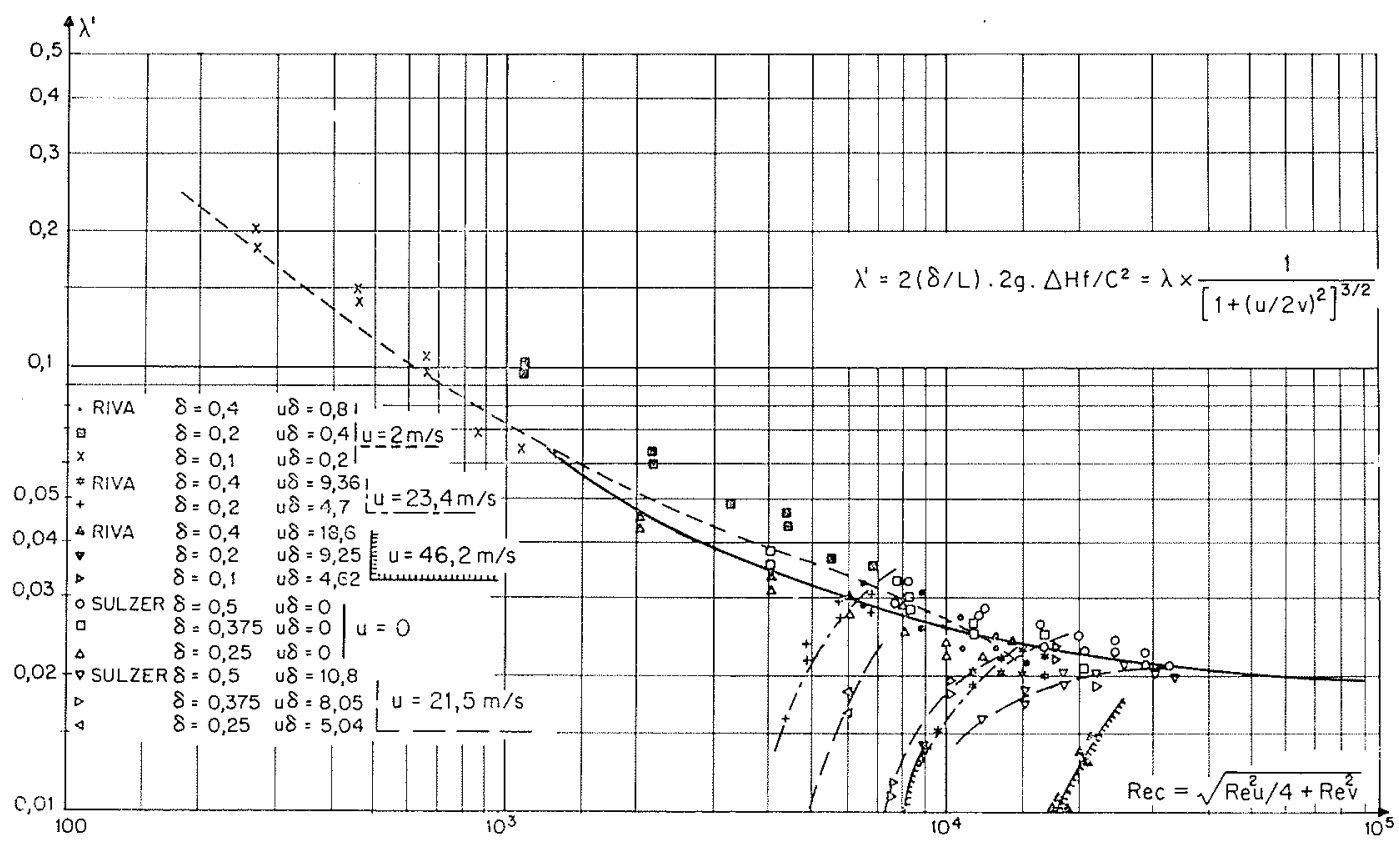

11.21

$\lambda^{\prime}=f(\operatorname{Rec})\left(1^{\text {cr }}\right.$ schéma $)$

Courbes $\mathbf{U}=$ Cte.

$\lambda=\mathrm{f}($ Rec $)$ (first set-up)

Curves $U=C t e$.
Quand le joint tourne, la droite représentative des débits de fuite aurait une pente très légèrement inférieure à celle relevée pour une vitesse de rotation nulle.

Des deux courbes ci-dessus il faut retenir que le débit de fuite expérimental, pour des valeurs industrielles de l'excentricité relative (savoir 0,3 en moyenne) est systématiquement inférieur au débit de fuite calculé.

Partant de la figure II.1 (extraite de Impeller Pumps, travaux d'Egli), on peut établir que pour une valeur de $\mathrm{L} / 2 \delta$ de l'ordre de 200 (ce qui est une valeur industrielle) et pour des excentricités relatives variant entre 0 et 1 , l'augmentation du débit de fuite est au maximum de $12 \%$ pour un jeu de $0,07 \mathrm{~mm}$ et de $20 \%$ pour un jeu de $0,2 \mathrm{~mm}$.

Ce dernier résultat est à rapprocher de l'affirmation de Cornish [11], selon laquelle l'augmentation du débit de fuite (régime turbulent) due à l'excentricité est inférieure à $30 \%$.

\section{Conclusion :}

Pour des valeurs industrielles de l'excentricité relative, le rapport $Q_{\text {excentré }} / Q_{\text {eentré }}$ pourrait varier de 1 à 1,15 ou 1,20 . Ce fait pourrait expliquer la dispersion de certains résultats expérimentaux et met en relief l'importance du paramètre excentricité, malheureusement très difficile à prévoir et à contrôler.

\section{Schémas possibles de l'écoulement dans le joint lisse}

La détermination du débit de fuite dans les labyrinthes cylindriques lisses nécessite l'utilisation de paramètres spécifiques d'un certain mode d'écoulement.

On peut définir plusieurs formes d'écoulement faisant intervenir des paramètres différents, mais il est préférable de ne retenir que celles qui font appel à des notions connues, et à des développements relativement simples.

Deux formes d'écoulement sont à retenir.
Premier schéma : écoulement hélicoïdal.

Une particule de fluide se déplaçant dans le joint est soumise à deux composantes de vitesse :

— vitesse axiale : $\mathrm{V}$;

- vitesse tangentielle : $u$.

Elle suit donc une trajectoire hélicoïdale.

Ce schéma fait apparaître les paramètres suivants :

- vitesse absolue de la particule $\mathrm{C}$ qui est la résultante de $u$ et de V;

- une trajectoire de longueur moyenne $L^{\prime}$;

- un coefficient de perte de charge $\lambda^{\prime}$ le long de la trajectoire;

- un nombre de Reynolds absolu $\operatorname{Re}_{c}$ représentatif de l'écoulement.

Calcul de $C$ (vitesse absolue).

Il est possible de définir $\mathrm{C}$ de façon analytique à partir des fonctions $u=f(\mathrm{Y})$ et $\mathrm{V}=g(\mathrm{Y})$. Mais $u$ et $\mathrm{V}$ sont fonction de la nature de l'écoulement (laminaire ou turbulent) et, s'il est facile de déterminer $\mathrm{V}=\mathrm{g}(\mathrm{Y})$, il est plus difficile de définir $u=f(\mathrm{Y})$.

Le calcul de $\mathrm{C}$ ne sera donc fait qu'en fonction des composantes moyennes de vitesse (schéma ci-dessous).
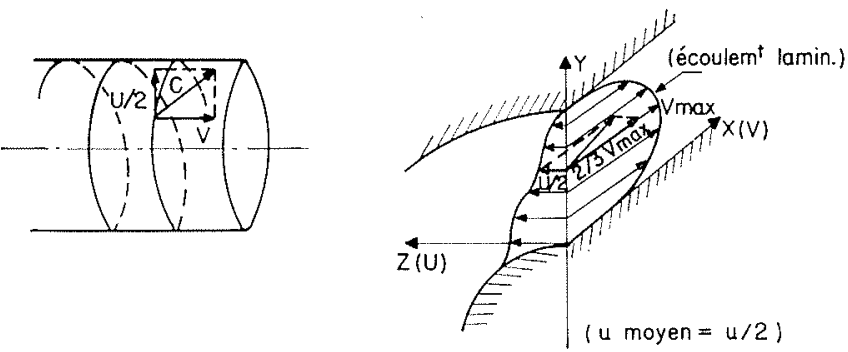

L'écoulement tangentiel que nous avons représenté sera défini d'après Prandtl (travaux de Taylor). 
Composantes moyennes de la vitesse $C$.

- composante tangentielle: $u / 2$;

- composante axiale: V.

D'où :

$$
C=\eta V \quad \text { avec } \quad \eta=\sqrt{1+(u / 2 V)^{2}}
$$

Calcul de la trajectoire (longueur $\mathrm{L}^{\prime}$ ).

Soit :

$L^{\prime}=$ longueur moyenne de la trajectoire hélicoïdale;

$\mathrm{L}=$ longueur moyenne de la trajectoire circulaire;

$\mathrm{L}^{\prime}=\eta \mathrm{L}$.

Calcul du nombre de Reynolds absolu $\mathrm{Re}_{e}$.

$$
\operatorname{Re}_{c}=2 \delta \mathrm{C} / \nu=\left[0,25\left(\operatorname{Re}_{u}\right)^{2}+\left(\operatorname{Re}_{v}\right)^{2}\right]^{1 / 2}
$$

Calcul de $\lambda^{\prime}$.

$$
\lambda=2(\delta / \mathrm{L}) 2 g \Delta \mathrm{H} f / \mathrm{V}^{2}
$$

$(\Delta \mathrm{H} f=$ chute de pression due aux fuites).

$$
\lambda^{\prime}=2\left(\delta / L^{\prime}\right) 2 g \Delta H f / C^{2}
$$

d'où :

$$
\lambda^{\prime}=\lambda / \eta^{3}
$$

En utilisant le diagramme de la figure $I .4\left[\lambda=f\left(\operatorname{Re}_{\mathrm{V}}\right)\right]$, nous avons tracé (fig. II.2) les courbes $\lambda^{\prime}=f\left(\operatorname{Re}_{c}\right)$ connaissant $\lambda, u, \operatorname{Re}_{u}, \operatorname{Re}_{\mathrm{Y}}$, etc.

Les résultats sont assez décevants, du moins lorsque l'on recherche une loi générale et unique. Dans ce cas on constate une dispersion relativement grande des points, notamment pour des vitesses périphériques importantes. Ceci est dû au fait que les approximations envisagées pour le calcul de la vitesse absolue sont trop importantes.

Il est certain que si celle-ci était connue avec plus de précision, on obtiendrait des réseaux de courbes à $u=$ Cte.

Par ailleurs, nous avons négligé dans la détermination de C l'influence de la force d'inertie centrifuge qui agit radialement sur chaque particule fluide.

L'écoulement tri-dimensionnel devient de ce fait encore plus complexe.

En conclusion, la complexité des calculs et la nature de la documentation actuelle ne permettent pas une interprétation utile et valable des pertes par fuite dans les labyrinthes lisses par la considération d'un schéma d'écoulement hélicoïdal. Nous l'abandonnerons donc pour le moment.

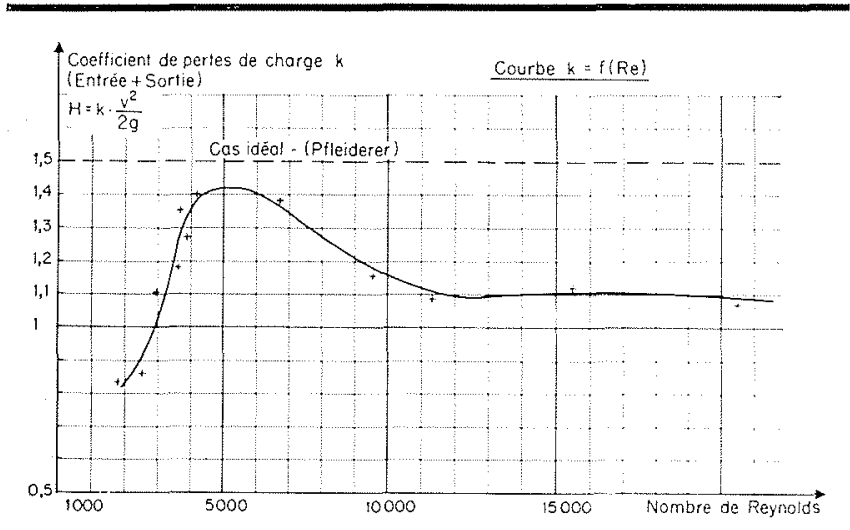

II.3/ Courbe représentative du coefficient de pertes de charge à l'entrée et à la sortie du joint lisse pour l'air. Laboratoire Hydraulique ENSAM (Paris 1963).

Typical curve showing coefficient of head losses at the inlet and outlet of a smooth seal for air. ENSAM Hydraulic Laboratory (Paris, 1963).
Deuxième schéma : écoulement axial + paramètres faisant iniervenir la rotation du tambour.

La vitesse de rotation n'ayant qu'une influence limitée (voir à cet effet la figure II.5), sur le débit de fuite, elle n'apparaîtra que par l'intermédiaire de coefficients de correction mathématiques ou physiques (c'est notamment le cas des travaux de M. Viano et de Franco Tosi).

Les paramètres principaux utiliseront alors les caractéristiques de l'écoulement axial.

\section{Formule de base utilisée (voir également nota).}

La relation de base servant pour les différents calculs est la formule de Pffeiderer qui a été reprise par d'autres auteurs tels que: Stepanoff, De Kowats et Desmur, Sedille, Prandtl.

Cette relation est la suivante:

$$
\Delta \mathrm{H}=\lambda \frac{\mathrm{L}}{2 \delta} \frac{\mathrm{V}^{2}}{2 \mathrm{~g}}+1,5 \frac{\mathrm{V}^{2}}{2 \mathrm{~g}}
$$

\section{Remarque.}

Le coefficient de perte de charge singulière à l'entrée du joint, posé égal à $0,5, \mathrm{~V}^{2} / 2 \mathrm{~g}$, ne prend cette valeur que dans le cas extrême où l'arête d'entrée du joint est idéalement vive (c'est-à-dire sans aucune bavure), et où la conduite amont a un diamètre très supérieur au niveau du raccordement avec le joint lisse.

La moindre éraflure ou bavure sur l'arête d'entrée du joint devient vite, à l'échelle du jeu (quelques dixièmes...) un véritable chanfrein qui, jouant le rôle de "tuyère 》, facilite alors l'écoulement du fluide (sa mise en vitesse) et abaisse de ce fait le coefficient de perte cinétique.

A la sortie du joint, le coefficient de perte de charge est égal à $1 . \mathrm{V}^{2} / 2 \mathrm{~g}$.

A l'école supérieure d'Arts et Métiers, une étude expérimentale relative à ce problème des pertes singulières a été réalisée sur une maquette soumise à un écoulement d'air.

Des mesures donnant les valeurs du coefficient de perte de charge cinétique (entrée + sortie), en fonction du nombre de Reynolds de l'écoulement axial à travers un joint ont permis de tracer la courbe $k=f$ (Re) (fig. II.3).

L'examen de cette courbe montre que ce coefficient, après avoir atteint la valeur maximale de 1,4, semble tendre asymptotiquement vers la valeur 1,1 .

Une étude expérimentale complémentaire a montré que ces résultats étaient valables pour l'eau.

Nota: M. Petermann néglige le terme $k \mathrm{~V}^{2} / 2 \mathrm{~g}$, qui a en fait peu d'influence sur $\mu$; il se traduit par une variation en moyenne de $5 \%$ sur la valeur de $\lambda$.

Si cette remarque a un intérêt théorique certain, il faut noter qu'industriellement il n'est pas nécessaire de connaître avec précision la valeur du coefficient de perte de charge singulière, car elle est très inférieure à la valeur du coefficient de perte de charge à travers le joint.

Ainsi, pour une turbine Francis ayant un $\mathrm{L} / 2 \delta$ égal à 70 , et pour un coefficient $\delta$ égal à 0,05 , une variation de $1 / 10^{\circ}$ du coefficient de perte de charge singulière entraine une variation de $\Delta \mathrm{H}$ de lordre de $3 / 100$.

En ce qui concerne les travaux du groupe de travail de M. Viano et ceux de M. Petermann, une variation de $1 / 10^{\circ}$ du coefficient de perte de charge singulière entraîne une variation de $\Delta \mathrm{H}$ de lordre de 1/100.

En conclusion, le coefficient de perte de charge singulière global $1,5\left(\mathrm{~V}^{2} / 2 g\right)$ étant trop élevé, la valeur $I, I$ à 1,2, qui correspond beaucoup mieux à la réalité, sera retenue. 


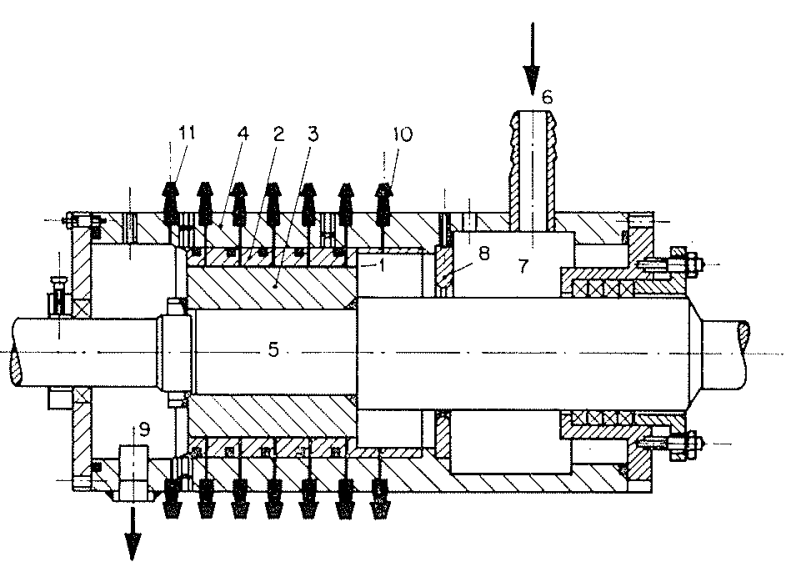

II.4/ Section de l'appareil expérimental utilisé par M. Petermann pour ses cssais relatifs au débit de fuite dans les joints lisses.

1. Joint lisse. / 2 et 3. Manchons en cuivre délimitant le joint. / 4. Stator sur lequel est monté le manchon 2. / 5. Arbre ou rotor sur lequel est monté le manchon 3. I 6 et 9. Tuyau d'arrivée d'eau (6), et de sortie (9). / 7. Chambre d'arrivee d'eau. / 8. Diaphragme de stabilisation d'arrivée d'eau. / 10 et 11. Prises de pression d'entrée (10) et de sortie (11).

Cross-section through apparatus used by Petermann in tests on leakage through smooth seals.

1. Smooth seal. / 2-3. Copper sleeves limiting the seal. / 4. Stator bearing sleeve 2. 15 . Shaft or rotor bearing sleeve 3. I 6-9. Water inlet pipe (6) and water outlet pipe (9). /7. Water inlet chamber. / 8. Water inlet stabilizing diaphragm. / 10-11. Inlet (10) and outlet (11) pressure tappings.

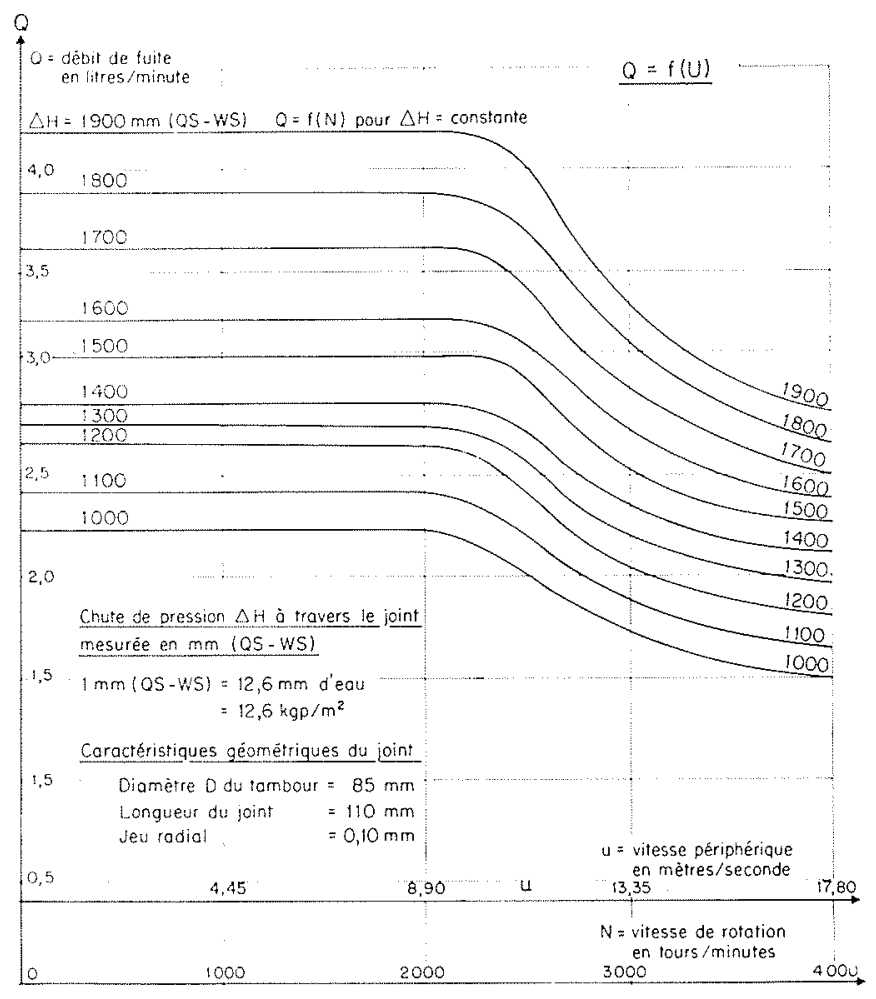

II.5/ Débit de fuite à travers un joint lisse en fonction de la vitesse de rotation pour un $\Delta H$ constant (expérience de M. Petermann).

Leak flow through a smooth seal versus rotation speed at constant $\triangle H$ (Petermann's experiment).

\section{Etude comparée des travaux du groupe de travail de M. Viano et des travaux de M. Petermann}

Après avoir apporté quelques compléments à l'étude du groupe de travail de M. Viano, nous nous proposons l'analyse des travaux de M. Petermann, et leur association aux précédents.

\section{Compléments à l'étude du groupe de travail de M. Viano.}

Rappelons que cette étude conduit à tracer un réseau de courbes (fig. I.6) permettant de déterminer le début de fuite connaissant la différence de pression entre l'entrée et la sortie du labyrinthe et les caractéristiques de ce dernier. En abscisse est porté un coefficient $\operatorname{Re}^{\prime}{ }_{y t}$ qui, dans le cas des grosses turbines industrielles, prend la forme simplifiée $(5000 \sqrt{2 g \Delta \mathrm{H})} / \mathrm{U}$, qui présente l'intérêt de rester constante pour tous les fonctionnements homologues de machines semblables.

La zone de la figure I.6 intéressant les pompes et les turbines est relativement limitée et serait à explorer en priorité lors de nouveaux essais.

Calculons par exemple les limites de cette zone dans le cas des pompes.

- L'énergie totale à la sortie de la roue est égale à :

$$
\mathrm{H}=\eta_{r}\left(u_{2}^{2} / g\right) m \quad \text { avec } \quad m=\mathrm{V} u_{2} / u_{2}
$$

- L'énergie cinétique relative est sensiblement égale à $0,5 \mathrm{~m}$.

On en déduit que la différence de pression entre l'entrée et la sortie de la roue est :

et :

$$
\Delta \mathrm{H}=\mathrm{H}(1-0,5 m)
$$

$$
\sqrt{2 g \Delta \mathrm{H} / u_{1}}=\left(u_{2} / u_{1}\right) \sqrt{2 \eta_{1} m(1-0,5 m)}
$$

Les valeurs extrêmes approximatives de cette expression sont, en admettant $\eta=1$ :

- pour les faibles valeurs du $n_{\mathrm{S}}$ :

$$
u_{2} / u_{1}=1,7 \quad m=0,55 \quad \sqrt{2 g \Delta \mathrm{H} / u_{1}}=1,52
$$

- pour les grandes valeurs du $n_{\mathrm{S}}$ :

$$
u_{2} / u_{1}=1,3 \quad m=0,3 \quad \sqrt{2 g \Delta \mathrm{H} / u_{2}}=0,93
$$

Donc, si $\Delta H$ était la différence de pression entre la sortie et l'entrée de la roue, les valeurs extrêmes de $\operatorname{Re}^{\prime}{ }_{\nu t}$ seraient 7600 et 4600 . Mais le $\Delta \mathrm{H}$ pris en considération est la différence de pression de part et d'autre du labyrinthe. Cette valeur est toujours nettement inférieure à la différence de pression entre grand et petit diamètre de la roue et, de ce fait, les valeurs extrêmes de $\operatorname{Re}_{w i}^{\prime}$ données précédemment doivent être réduites.

Cette remarque semble donner une explication valable au fait que tous les points rapportés sur la figure 1.6 se groupent de telle manière que $3500<\operatorname{Re}_{y: 1}^{\prime}<6000$. Il y a lieu de rappeler que ces points sont relatifs à des installations industrielles exécutées par la maison Riva; les valeurs de $\Delta \mathrm{H}$ ont été calculées par la formule:

$$
\Delta \mathrm{H} / \mathrm{H}=1-\mathrm{K}^{2}{ }_{11}+\mathrm{K}^{2}(2)+0,25 \mathrm{~K}^{2}{ }_{112}-0,25 \mathrm{~K}^{2}{ }_{11}
$$

Dans les machines modèles le terme $l$ n'est plus négligeable devant le terme $2 \delta u / 5000 \mathrm{v}$.

M. Viano n'a pas pris en considération l'influence de 
l'excentricité sur le débit de fuite, ainsi que celle de la rugosité des parois du joint.

\section{Etude des travaux de M. Pelermann.}

A) Il est tout d'abord nécessaire d'introduire dans cette étude le compte rendu du rapport de Brïnswick de 1966 relatif à l'influence de la vitesse de rotation sur le débit de fuite dans des jeux cylindriques. Ces travaux ont été réalisées par MM. B. Stampa, M. Pekrun et H. Petermann (Pfleiderer-Institut für Strömungsmaschinen, Technische Hochschule Braunschweig, Germany).

\section{Introduction :}

A l'Institut Pffeiderer, le travail de recherche est poursuivi dans le seul but de fournir des données permettant le calcul des labyrinthes lisses des pompes centrifuges à eau. Il est donc nécessaire d'établir les coefficients de frottements et la forme des écoulements dans ces anneaux cylindriques. Les résultats de ces expériences ont été jusqu'à maintenant comparés et insérés dans les études connues relatives à ce sujet.

\section{Expériences faites au Pfleiderer Institute:}

- Description de l'appareil expérimental (voir fig. II.4);

- Ces expériences ont été commencées en 1964 et sont actuellement poursuivies.

\section{Données expérimentales:}

- longueur $\mathrm{L}$ du joint lisse : $\mathrm{L}=110 \mathrm{~mm}$;

- diamètre du tambour: $\mathrm{D}=65-85-110 \mathrm{~mm}$;

- jeux radiaux $=0,1-0,15-0,20 \mathrm{~mm}$;

- chute de pression et débit de fuite mesurés avec des vitesses de rotation variant entre 0 et $4000 \mathrm{tr} / \mathrm{mn}$.

Remarque: Difficulté pour réaliser un anneau à la fois étroit et co-axial.

\section{Résultats et discussions:}

La figure II.5 montre la variation de débit $Q$ en $1 / \mathrm{mn}$ en fonction de la vitesse de rotation $\mathrm{N}$ ou de la vitesse périphérique $u$ en $\mathrm{m} / \mathrm{s}$, pour une chute de pression constante et les paramètres géométriques suivants:

$$
\begin{aligned}
\mathrm{L} & =110 \mathrm{~mm} \\
\mathrm{D} & =85 \mathrm{~mm} \\
\text { jeu radial } & =0,10 \mathrm{~mm}
\end{aligned}
$$

\section{Remarque:}

Les diagrammes faits pour des joints géométriquement différents ont la même forme générale.

a) Le trait caractéristique de tous ces diagrammes est la décroissance importante de débit pour une certaine gamme de vitesse (à partir de 2000 à $3000 \mathrm{tr} / \mathrm{mn}$ ).

b) Le nombre représentant l'écoulement est le nombre de Reynolds défini pour la vitesse axiale $\mathrm{V}$ :

$$
\operatorname{Re}_{i}=2 \mathrm{~V} . \delta / \nu(1)
$$

et pour la vitesse périphérique $u$ :

$$
\operatorname{Re}_{u}=2 u . \delta / \nu
$$

c) Le coefficient de frottement du fluide dans l'anneau est celui de la formule de Pffeiderer:

$$
\lambda=\frac{2 \delta}{\mathrm{L}} \cdot \frac{2 g \Delta \mathrm{H}_{i}}{\mathrm{~V}^{2}}
$$

On trouvera figure II.6 la transposition des résultats de la figure II.5 auxquels ont été adjoints d'autres résultats expérimentaux avec $\mathrm{L} / 2 \delta$ différents. On peut donc considérer

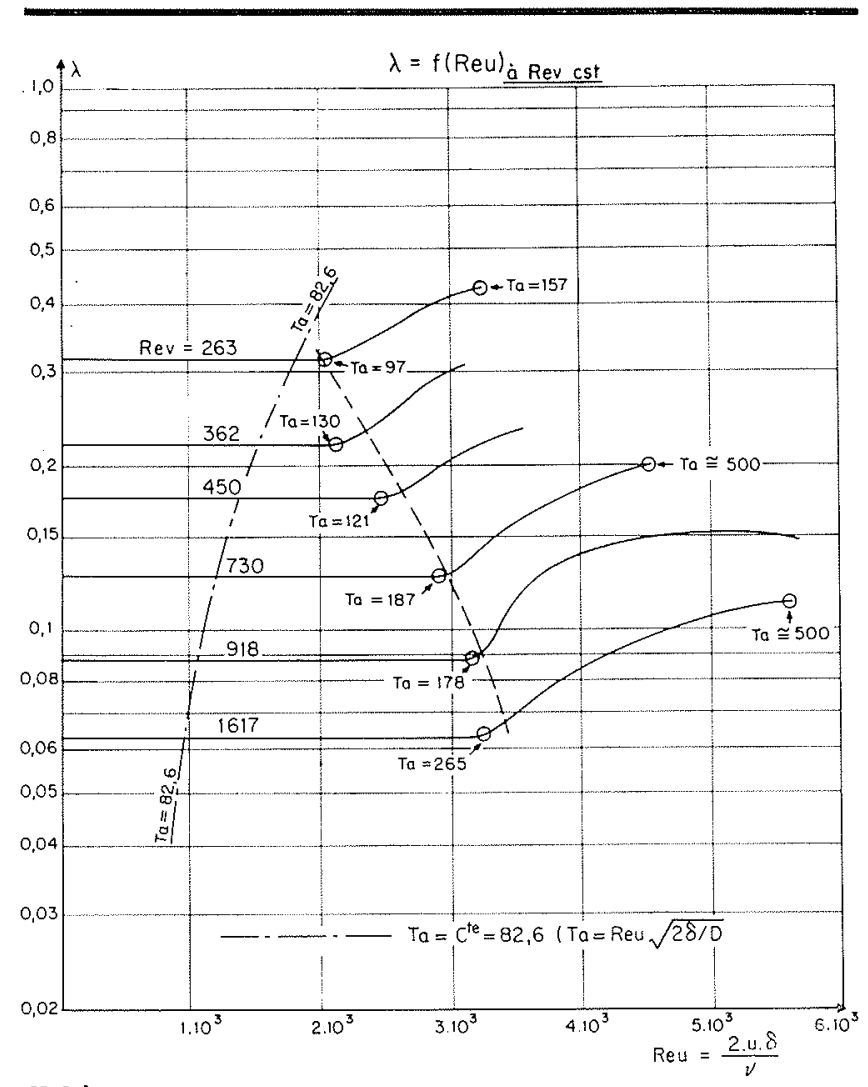

II.6/

qu'à notre connaissance, la figure II.6 résume les travaux de M. Petermann.

d) Le diagramme $\lambda=f\left(\operatorname{Re}_{u}\right)$ à $\operatorname{Re}_{i}=$ Cte figure II.6, montre que pour une certaine vitesse et un nombre de Reynolds axial $\operatorname{Re}_{v}$ constant, il y a accroissement subit du coefficient de frottement $\lambda$.

Le nombre de Reynolds axial $\mathrm{Re}_{v}$ restant toujours inférieur à 2000 , l'écoulement reste laminaire.

e) Trunovsky donne l'expression suivante pour l'écoulement laminaire dans un anneau:

$$
\lambda=\frac{96}{\operatorname{Re}_{v}}
$$

Cette formule appliquée aux résultats expérimentaux de la figure II.6 montre qu'elle n'est valable que pour des $\operatorname{Re}_{u}$ petits, c'est-à-dire pour des vitesses périphériques petites.

f) Le changement de forme de l'écoulement est lié au grand accroissement du coefficient de frottement; cette remarque a été nettement établie par les travaux de Taylor.

$$
\mathrm{Ta}=\mathrm{Re}_{n} \sqrt{\frac{2 \delta}{\mathrm{D}}}
$$

Il y a lieu de rappeler la définition du nombre de Taylor : (Cette définition du nombre de Taylor est tirée de Prandtl et c'est celle qui a été adoptée dans le présent document.) Taylor a fait apparaître l'existence d'un nombre de Taylor critique :

$$
\mathrm{Ta}=82,6
$$

Le changement de forme de l'écoulement est la limite entre l'écoulement laminaire et l'écoulement laminaire avec tourbillons de Taylor.

Au-delà de cette limite aucune équation complète pas plus théorique qu'empirique n'a pu être trouvée dans la littérature. 
R. HUGUENIN, P. BOUILLAUD et F. BREUIL

\section{Analyse des travaux de M. Petermann et association de ces travaux à ceux de M. Viano (Riva-Sulzer)}

Pour M. Petermann les paramètres de l'écoulement sont: - la vitesse axiale $\mathrm{V}$;

- le nombre de Reynolds axial $\operatorname{Re}_{v}=2 \mathrm{~V} \delta / \mathrm{V}$;

- le nombre de Reynolds circonférentiel : $\operatorname{Re}_{u}=2 \delta u / v$.

Le coefficient de frottement $\lambda$ a été défini par la formule de Pfleiderer simplifiée [terme $1,5 \mathrm{~V}^{2} / 2 \mathrm{~g}$ négligé].

INFLUENCE DE LA VITESSE DE ROTATION SUR LE DÉBIT DE FUITE (discussion sur figure II.5 : travaux de Petermann).

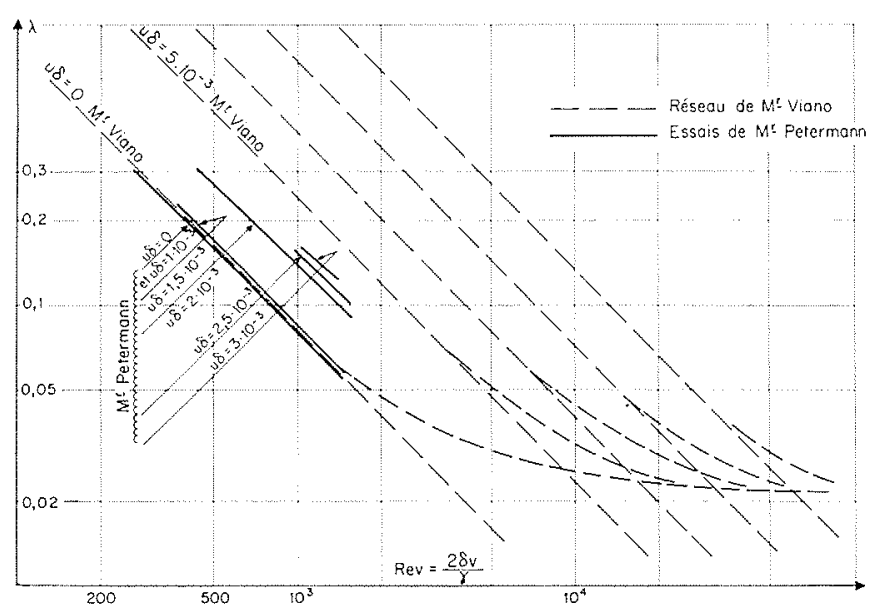

II.7/

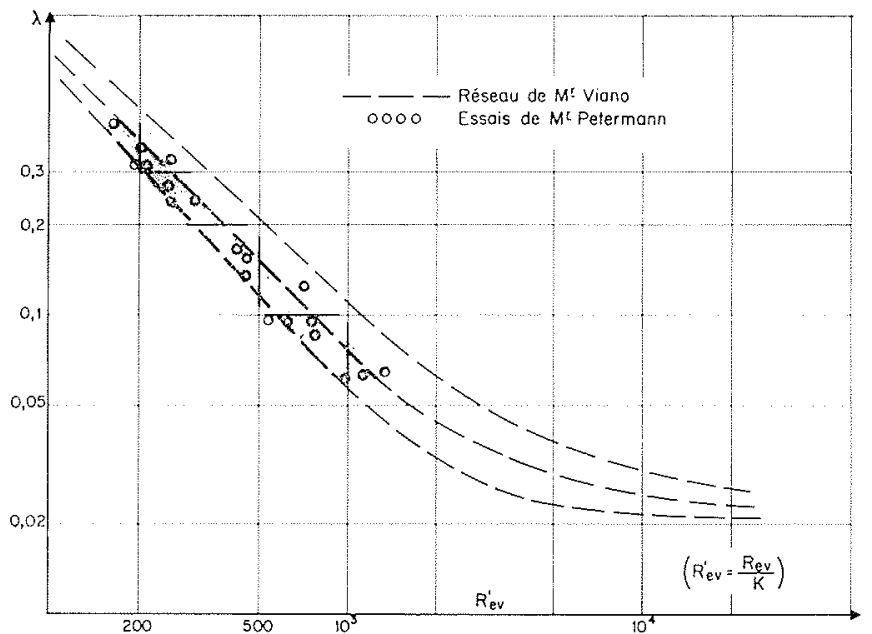

$11.8 /$

Ce graphique illustre très bien le fait que le débit de fuite est indépendant de la vitesse de rotation au-dessous de $2000 \mathrm{tr} / \mathrm{mn}(u=9 \mathrm{~m} / \mathrm{s})$. Au-dessus de cette vitesse critique le débit décroît et à $4000 \mathrm{tr} / \mathrm{mn}$ on remarque qu'il a diminué d'environ $30 \%$ pour chacune des courbes à chute de pression $\Delta \mathrm{H}=$ Cte.

INTRODUCTION DU NOMBRE DE TAYLOR DANS LES ESSAIS de M. Petermann:

Graphique $n^{\circ}$ II.6: Ce graphique, établi pour différentes valeurs de $L / 2 \delta$, est lié en partie au précédent et montre la croissance de $\lambda$ pour une certaine valeur de la vitesse de rotation, c'est-à-dire de $\operatorname{Re}_{w}$.

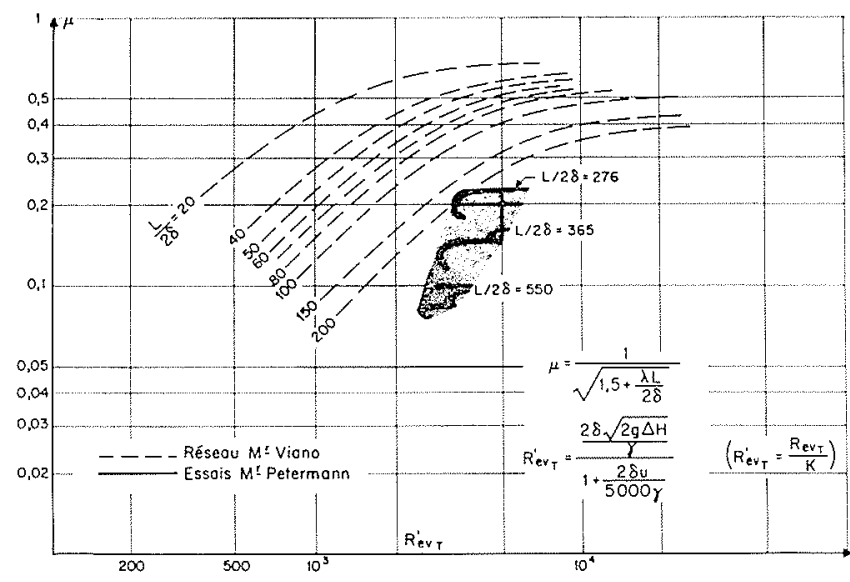

$11.9 /$

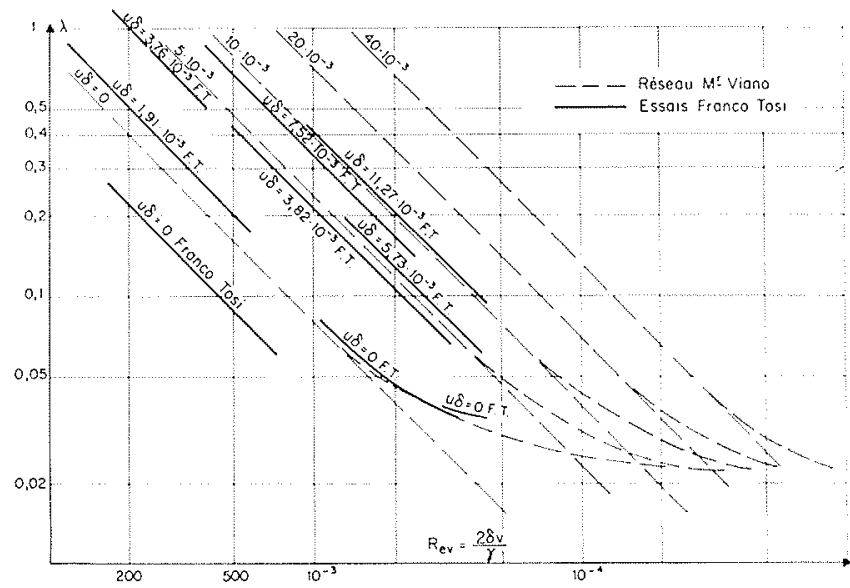

II.10/

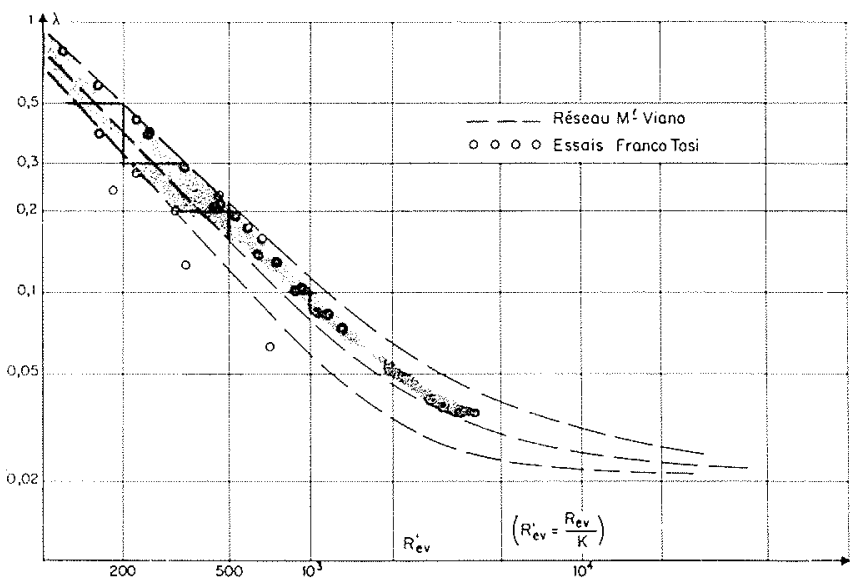

II.11/

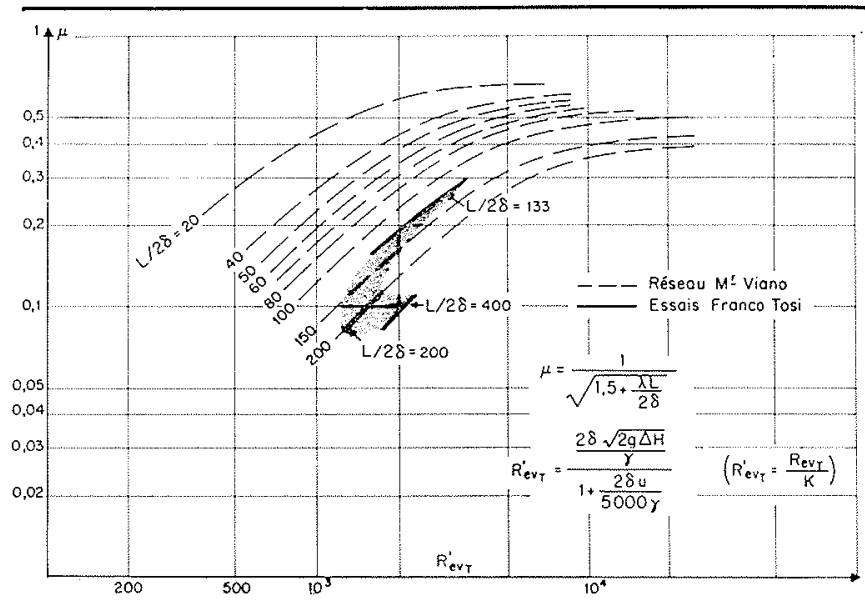

$11.12 /$ 
Sur les courbes paramétriques à $\mathrm{Re}_{v}=$ Cte :

- on a pointé le nombre de Taylor critique $\mathrm{Ta}=82,6$; - on a coté le point de transition en nombre de Taylor.

Trois remarques s'imposent:

a) Le point de transition s'écarte d'autant plus du nombre de Taylor critique que l'écoulement axial s'effectue à un nombre de Reynolds ( $\operatorname{Re}_{1}$ ) élevé.

b) La courbe $\mathrm{Ta}=82,6$ se rapproche d'autant plus $\mathrm{du}$ point de transition que $\operatorname{Re}_{v}$, est plus petit.

Cette dernière remarque nous amène à citer les travaux de Goldstein (1933) qui constate :

Pour un $\mathrm{Re}_{v}$, nul, le point de transition est en accord avec le nombre de Taylor.

Quand $\mathrm{Re}_{v}$ passe de 0 à 31 , la vitesse critique augmente de $20 \%$, puis elle diminue rapidement quand $\mathrm{Re}_{v}$ passe à 52 .

Pour les grandes valeurs de $\operatorname{Re}_{v}$ Goldstein indique que la vitesse critique calculée est passablement fausse.

c) L'expression :

$$
\operatorname{Re}_{v t}^{\prime}=\frac{\operatorname{Re}_{1 t}}{1+\operatorname{Re}_{u} / 5000}
$$

résultant des essais de M. Viano, peut s'écrire:

$$
\operatorname{Re}_{1 t t}^{\prime}=\frac{\operatorname{Re}_{1 \dot{r}}}{1+\frac{\mathrm{Ta} \sqrt{(\mathrm{D} / 2 \delta)}}{5000}}
$$

cette dernière expression montrant la relation entre $\operatorname{Re}_{v t}^{\prime}$ et Ta.

Implicitement la notion expérimentale de $\mathrm{Re}^{\prime}$, montre que le nombre de Taylor a besoin d'être affecté d'un coefficient géométrique.

Ces trois remarques montrent que le nombre de Taylor n'est pas un paramètre suffisant pour analyser les écoulements industriels caractérisés par des grands $\operatorname{Re}_{v}$ et des grands $\operatorname{Re}_{u}\left(\operatorname{Re}_{v}\right.$, variant de 15000 à $30000, \operatorname{Re}_{u}$ variant de 40000 à 80000 et Ta pouvant atteindre la valeur de 3600 ).

Figure II.7 (correspondant à la figure I.4 de la première partie). Nous avons porté avec les mêmes échelles les résultats de M. Petermann sur le diagramme de M. Viano (courbes à $u \delta=\mathrm{Cte}$ ). Les résultats de $\mathrm{M}$. Petermann cadrent avec ceux de M. Viano.

Figure II.8 (correspondant à la figure I.5). Les mêmes points reportés avec la même abscisse montrent un « tassement $\gg$ des mesures, comme l'avait déjà constaté $M$. Viano.

Figure II.9 (correspondant à la figure I.6). Les mêmes points « dépouillés » en $\mu$ s'insèrent dans Je réseatı « extrapolé » de M. Viano.

Remarques sur les figures $11.7,11.8$ et 11.9 : mann.

Elles définissent la zone d'expérimentation de M. Peter-

Il résulte de l'examen général que $M$. Petermann :

a travaillé à des $\mathrm{L} / 2 \delta$ très élevés par rapport aux autres expérimentateurs;

- a travaillé à des $\operatorname{Re}_{v t}^{\prime}$ un peu faibles.

\section{Analyse des essais \\ Franco Tosi et association de ces essais à ceux de M. Viano}

Franco Tosi a adopté des définitions et des paramètres semblables à ceux employés par M. Viano (Riva - Sulzer).

a) Ces essais se sont traduits finalement par le graphique n" II.10: $\lambda=f\left(\operatorname{Re}_{\mu}\right)$ à $u \delta=$ Cte.

La zone d'expérimentation est moins vaste que celle de Riva-Sulzer, mais les courbes Franco Tosi s'insèrent correctement dans le réseau de M. Viano (à l'exception toutefois de certaines courbes $\delta=0,1$ en $u \delta=0$ : cette divergence aux petits jeux peut s'expliquer par l'influence de l'excentricité dont l'influence en valeur relative devient maximale).

b) Le graphique $\mathrm{n}^{\circ}$ II. 11 est la traduction des essais précédents : $\lambda=f\left(\operatorname{Re}_{v}^{\prime}\right)$.

On assiste à un «tassement » des points de mesure.

c) Le graphique $n^{\circ}$ II.12 traduit en $\mu=f\left(\operatorname{Re}^{\prime}{ }_{t t}\right)$ les essais de Franco Tosi; ces résultats s'insèrent parfaitement dans le réseau de $\mathrm{M}$. Viano.

\section{Conclusions}

Bien que situés en grande partie dans une zone différente, les résultats de $M$. Petermann cadrent avec ceux de M. Viano.

Etant donné que dans les machines industrielles $\operatorname{Re}_{v}$ varie de 15000 à 30000 et $\operatorname{Re}_{u}$ de 40000 à 80000 , il faut conclure que les essais de M. Petermann sont éloignés des conditions industrielles.

Situés dans la même zone d'expérimentation, les résultats des essais de Franco Tosi concordent presque en intégralité avec ceux de M. Viano.

M. Viano a fait apparaître un terme de similitude $\sqrt{2 g \mathrm{H}} / u$ et grâce à un artifice mathématique (coefficient K) a obtenu un "tassement » des résultats des mesures aussi bien pour les essais de M. Petermann que pour ceux de Franco Tosi.

Le paramètre $\mathrm{Re}^{\prime}{ }_{t t}$ est donc un paramètre valable, qui a en outre l'avantage de permettre un calcul direct et facile du débit de fuite.

Les écarts, avec le calcul industriel préconisé, peuvent être au maximum de $10 \%$.

Ces écarts peuvent être imputables en partie au paramètre excentricité dont l'influence est mal connue et difficilement contrôlable.

\section{Bibliographie générale}

Prleiderer: Pompes centrifuges. (Dumod).

DE Kowats et Desmur: Pompes-ventilateurs-compresseurs centrifuges et axiaux. (Dunod).

STEPANOFf: Pompes centrifuges et hélices. (Dunod).

LAZARKIEWICZ et A. Troskolanski: Impeller pumps. (Pergamon Press)

Prandte: Mécanique des fluides.

Petermani : Compte rendu du Congrès de Brunswick 1966

Franco Tosi : Essais sur labyrinthes lisses.

[1] BECKER (E.). - Stroemungsvorgaenge in ringfoermigen Spalten Z. VDI., 51 (1907), p. 1133.

[2] Just (K.). - Ueber Labyrinthdichtungen fuer Wasser. Diss. TH. Darmstadt $(1910)$ 
[3] WINKEL (R.). - Die Wasserbewegung in Leitungen mit Ringspalt Durchflussquerschnitt. ZAMM, 3 (1923), p. 251.

[4] Sozuki (S.). - On the Leakage of Wather through Clearance Space, Joumal of the Fac. Eng. Tokyo, Imp. Univ., 18, No. 2 (1929).

[5] Tenot (A.). - Turbines hydrauliques et régulateurs automatiques de vitesse. Eyrolles éditeur, Paris, 1930, vol. 1, p. 385.

[6] Schneckenderg (E.). - - Der Durchfluss durch Drosselspalte Z. VDI., 74 (1930), p. 485.

[7] Bodart (E.). - Calcul de la perte aux joints dans les turbomachines. Congr. Intern. de Méc. Gén., Liège, 3 (1930), p. 42.

[8] Schneckenierg (E.). - Der Durchfluss von Wasser durch konz. u. exc. zylindrische Drosselspalte mit u. ohne Ringnuten. $Z A M M, 11$ (1931), p. 27.

[9] LEA (F.C.) and TAdros (A. G.). - Flow of water through a circular tube with a central core and through rectangular ubes. Phil. Mag., 7, 11 (1931), p. 1235.

[10] Stepanoff (A. J.). - Leakage Loss and Axial Thrust in Centrifugal Pumps. Trans. ASME, 30-11-1931, 54 (1932), p. 65.

[11] Cornist (R. J.). - Flow of water through Fien Clearances with Relative Motion of the Boundaries. Proc. Roy. Soc., A 140 (1933), p. 227.

[12] WendT (F.). - Turbulente Stroemungen zwischen zwei rotierenden konaxialen Zylindern. Ing. Arch. IV (1933), p. 577.

[13] Piercy (N. A. V.), Hooper (M. S.) and Winny (H. F.). - Viscous Flow through Pipes with Cores. Phil. Mag., 7, 15 (1933). p. 647 .

[14] Gercke (M. J.). - Berechnung der Ausflussmengen von Labyrinthdichtungen. Waerme, 57 (1934), p. 513.

[15] Taylor (G. J.). - Fluid Friction Between Rotating Cylinders I. Torque Measurements. Proc. Roy. Soc. A 157 (1936), p. 546.

[16] TrurnovskI (K.). - Spaltdichtungen. $Z$. VDI., 83 (1939), p. 857.

[17] Stepanof (A.J.). - Centrifugal pump performance as a Function of Specific Speed. Trans. ASME, 65 (1943), p. 629.

[18] PFenninger (A.). - Bulletin Escher Wyss, tome XX, 1946-47, p. 31 et 32 .

[19] Fontaine (M.). - Quelques applications de la méthode thermométrique Poirson. La Houille Blanche (1951), p. 12.

[20] Fontaine (E.) el Campmas (P.). - Sur la séparation des pertes dans une turbine Francis. La Houille Blanche (1952), p. 269.

[21] Notbaar (R. F.) and Kintner (R. C.). - Fluid Friction in annuli of small clearance. Proc. Sec. Midw. Cont. Fluid. Mech. (1952), p. 185.

[22] Kovats (A. de) et Desmur (G.). - Pompes, ventilateurs, compresseurs centrifuges et axiaux. Dunod, Paris (1953).

[23] Pfleiderer (C.). - Die Kreiselpumpen fuer Fluessigkeiten und Gase Vierte Auflage. Springer Verlag, Berlin (1955).

[24] Bouquier (J.). - Exposé de quelques essais pratiques d'amélioration des labyrinthes de turbines Francis. V"s Journées de l'Hydraulique, Aix-en-Provence, juin 1958

[25] Ferry (S.), WILlm (G.) et Thouvenin (J.). - L'influence de l'usure sur le rendement des turbines hydrauliques. Ves Journées de l'Hydraulique, Aix-en-Provence, juin 1958.

[26] Gazley (C.). - Heat-Transfer Characteristics of the Rotational and axial Flow between Concentric Cylinders. Trans. ASME, 30 (1958), p. 79.

[27] Donelly (R. J.). - Experiments on the stability of viscous flow between rotating cylinders. 1. 1. Torque Measurements. Proc. Roy. Soc., A 246 (1958), p. 312 .

[28] Doneliy (R. J.) and Simon (N. J.). - An empirical torque relation for supercritical flow between rotating cylinders. $J$. Fluid. Mech., 7 (1960), p. 401.

[29] Viano (M.). - Essais en laboratoire pour la détermination des pertes dans les labyrinthes des turbines Francis. S.H.F. Symposium Nice 1960.

[30] Stepanoff (A.J.). - Pompes centrifuges et pompes hélices. Traduit par M. Hug. Dunod, Paris (1961).

[31] TrllmanN (W.). - Zum Reibungsmoment der turbulenten Stroemung zwischen rotierenden Zylindern. Forschg. Ing. Wes., 27 (1961), p. 189.

\section{Discussion}

M. le Président remercie vivement $M$. Viano et $M$. Huguenin de leurs exposés. Ils sont la concrétisation diun travail important, dont il y a lieu de souligner l'utilité pratique.

Jusqu'à présent, les formules habituelles proposées par certains auteurs pour le calcul des fuites dans les labyrinthes ne tenaient compte que de la vitesse axiale; la composante vitesse tangentielle de rotation n'intervient pas.
M. Viano nous propose non seulement une formule, mais des abaques très pratiques, faisant intervenjr l'influence de la vitesse tangentielle. Le dosage de cette influence est un résultat d'expérience et non l'aboutissement d'une théoric. Par contre, comme développé au cours de cette communication, les résultats obtenus par l'application des formules de $\mathrm{M}$. Viano coïncident bien avec les résultats d'essais obtenus par d'autres expérimentateurs.

D'ores et déjà, il est donc possible de disposer de formules pratiques plus précises que celles utilisées jusqu'ici.

Se référant à la deuxième partie du rapport, M. Zanetri (Société Riva-Calzoni) propose une expression thérique de $\lambda^{\prime}$ qui lui paraît interpréter plus fidèlement les résultats expérimentaux que celle proposée dans le rapport ci-dessus.

En effet, si on admet que l'écoulement dans le joint est équivalent à ce qui se passe entre parois immobiles, le fluide étant animé de la vitesse moyenne $C$, on peut écrire, avec les notations employées, que la sollicitation de frottement par unité de surface entre parois et fluide, a pour expression:

$$
\frac{\lambda^{\prime}}{4} \gamma \frac{\mathrm{C}^{2}}{2 g}
$$

( $\gamma=$ poids spécifique du fluide).

Il va de soi que cette sollicitation est dirigée dans le sens de la vitesse $C$. On a donc, pour l'équilibre, dans le sens axial, des forces de pression et de frottement agissant sur le joint :

$$
\pi \mathrm{D} \delta \gamma \Delta \mathrm{H}_{i}=\pi \mathrm{D} 2 \mathrm{~L} \frac{\lambda^{\prime}}{4} \gamma \frac{\mathrm{C}^{2}}{2 g} \frac{\mathrm{V}}{\mathrm{C}}
$$

d'où, en simplifiant et en utilisant aussi les notations du rapport susvisé :

$$
\lambda=2(\delta / L) 2 g \Delta H_{f} / V^{2}
$$

et en définitive :

$$
\lambda^{\prime}=\lambda \frac{1}{\left[1+(u / 2 \mathrm{~V})^{2}\right]^{11}}
$$

Les points $\left(\hat{\lambda}^{\prime}, \operatorname{Re}_{e}\right)$ obtenus par la relation (1) sont encore assez dispersés, mais dans une mesure moindre que ceux du diagramme présenté dans le rapport.

Les catuses de cette dispersion sont essentiellement les mêmes que celles citées par MM. Viano et Huguenis, et en particulier : l'approximation de la théorie, qui néglige, entre autres, l'effet des forces centrifuges, de l'excentricité, de la rugosité, etc.

Il semble opportun d'ajouter, pour mieux éclairer les limites des hypothèses théoriques adoptées, que les joints expérimentés, - comme du reste ceux des machines hydrauliques, - présentent un développement axial tellement limité que l'influence des valeurs du coefficient de frottement dans les différents points de la partie initiale du joint, où l'écoulement n'a pas encore atteint sa valeur de régime, est en général prépondérante par rapport à la valeur du même coefficient en régime établi.

En ce qui concerne, par exemple, la vitesse périphérique de l'eau dans le joint, il est possible de calculer - en tenant compte des efforts de frottement que l'on pent présumer entre paroi et fluide et de l'inertie de ce dernier, - que le temps de mise en régime est souvent supérieur à celui mis par l'eau pour traverser le joint.

Il s'ensuit, en particulier, que si l'eau se présente à l'entrée avec une vitesse périphérique négligeable - comme c'est le cas dans l'appareil employé pour les essais, - sa vitesse périphérique dans le joint reste en moyenne beaucoup plus faible que celle de régime $(u / 2)$ prise comme base des considerations théoriques.

En définitive, le problème de l'interprétation théorique du phénomène est très complexe, principalement en raison de la multiplicité des facteurs qui l'influencent et qui normalement ne sont pas connus a priori; il semble donc préférable, eu égard aux buts pratiques du travail analysé, de tirer des résultats d'essais une relation tout simplement empirique ainsi qu'il a été fait par les auteurs de la communication présentée.

M. Huguenin se déclare d'accord sur la première remarque présentée par M. ZANETT, mais il craint que le résultat des interpré tations théoriques ne reflète guère que les hypothèses qui ont dû être admises pour établir celles-ci.

M. le Président clôt la discussion en soulignant l'intérêt de l'intervention de M. ZaNerTr, qui met bien en lumière les difficultés de la mise sur pied actuellement d'une étude théorique satisfaisante; c'est la raison pour laquelle la «Section Machines 》 s'est bornée aujourd'hui à présenter des résultats expérimentaux, immédiatement utilisables, mais la poursuite de la discussion sur les points soulevés par M. ZanetTi sera reprise au sein de la Section. 